\title{
International Survey on the Management of Acute Kidney Injury in Critically III Patients: Year 2007
}

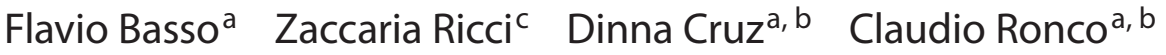

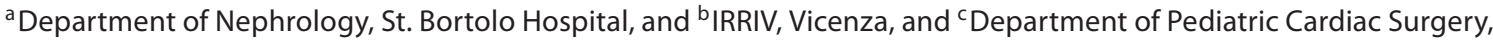 \\ Bambino Gesù Children's Hospital, Rome, Italy
}

\section{Key Words}

Critical care nephrology • Acute kidney injury •

Renal replacement therapy $\cdot$ Dialysis dose prescription

\begin{abstract}
Introduction: Several aspects of acute kidney injury (AKI) management, including medical approaches to AKI patients and the optimal form of renal replacement therapy (RRT), remain a matter of debate. Subjects and Methods: The responses of 440 participants to a questionnaire on several points of AKI management, submitted during the 4th International Course on Critical Care Nephrology in June 2007, were analyzed. Results: The most common answer to the definition of AKI was the use of the RIFLE criteria (55\%), followed by the presence of oligoanuria (24\%). Responders seemed to preferentially start dialysis within a creatinine range from $2.3-3.4 \mathrm{mg} / \mathrm{dl} \mathrm{(28 \% )} \mathrm{to} 3.4-4.5 \mathrm{mg} / \mathrm{dl}(26 \%)$ and with a urine output level of $150-200 \mathrm{ml} / 12 \mathrm{~h}$ (43\%). About $30 \%$ of responders showed that they would prescribe dialysis only in case of severe fluid overload (requiring mechanical ventilation and/or causing impaired skin integrity). Continuous RRT is used by most specialists $(86 \%)$, followed by intermittent hemodialysis (65\%), sustained low-efficiency dialysis (28\%) and peritoneal dialysis (30\%). The preferred RRT dosage was ' $35 \mathrm{ml} / \mathrm{kg} / \mathrm{h}$ ' (46\%) but $37 \%$ of responders did not explicitly answer this critical question. Bleeding, hypoten-
\end{abstract}

sion, filter clotting, vascular access and sepsis treatment were the most frequent complications and concerns of RRT. Conclusions: New classifications such as the RIFLE criteria did improve the well-known uncertainty about the definition of AKI. Awareness of the prescription and standardization of an adequate treatment dose seemed to have increased in recent years, even if there is still a significant level of uncertainty on this specific issue. Several concerns and RRT complications, such as bleeding and anticoagulation strategies, still need further exploration and development.

Copyright $\odot 2010$ S. Karger AG, Basel

\section{Introduction}

An intense epidemiological and clinical effort on acute kidney injury (AKI) recently led to a significant improvement in the consensus about the epidemiology, the classification and the approach to therapy of this complex syndrome [1]. The Acute Dialysis Quality Initiative (ADQI) [1,2] identified some years ago the critical areas where consensus was lacking: where possible, ADQI provided recommendations for good clinical practice, suggesting which clinical trials might have been necessary to generate an adequate level of evidence in this field. In this light, the RIFLE criteria (acronym indicating risk of renal dysfunction, injury to the kidney, failure of kidney func-

\section{KARGER}

Fax +4161306 1234 E-Mail karger@karger.ch www.karger.com
(C) 2010 S. Karger AG, Basel

0253-5068/10/0303-0214\$26.00/0

Accessible online at:

www.karger.com/bpu
Zaccaria Ricci

Department of Pediatric Cardiac Surgery

Bambino Gesù Children's Hospital

Piazza S. Onofrio 4, IT-00165 Rome (Italy)

Tel. +39 066859 2449, Fax +3906 6859 2670, E-Mail z.ricci@libero.it 
tion, loss of kidney function and end-stage kidney disease) for AKI definition were identified and successively revised $[1,3]$ : the application of these classifications to clinical practice showed how the acknowledgment of different levels of renal dysfunction can effectively identify clinical risk factors and eventually improve therapy and prevention [4]. Two most important clinical trials on the dose and prescription of renal replacement therapy (RRT) either in intermittent (Acute Renal Failure Trial Network, ATN) or continuous therapies (ATN and the Randomized Evaluation of Normal versus Augmented Level, RENAL) definitely established the adequate level of dialysis to be recommended in critically ill patients $[5,6]$. Recently, two important large surveys [the Dose Response Multicentre International collaborative Initiative (DoReMi) and the Beginning and Ending Supportive Therapy for the Kidney (BEST)] provided insightful information on AKI epidemiology, classification and therapy, RRT prescription delivery, timing, cost and mortality [7-12]. Nevertheless, the clinical practice of AKI management still shows a significant level of uncertainty and a lack of standard among many clinicians and operators. Following an observational practice-related approach, we asked the participants of an international course on critical care nephrology specific questions about practice patterns in AKI classification and therapy in order to verify whether routine clinical practice actually presents significant differences from what has been reported in the available literature.

\section{Subjects and Methods}

A questionnaire was widely distributed during the meeting and participants were invited to voluntarily complete the survey. Responses from participants who attended the Fourth Critical Care Nephrology International Survey held in Vicenza, Italy were surveyed. The questionnaire was a slightly modified version of the format used at the same meeting held 3 years previously [13]. Questionnaires were anonymous and completing them was not obligatory: participants were asked to share their individual experience and/or opinions. The form included 4 demographic questions and 17 multiple-choice questions and was divided into three sections. (1) The first section sought information about the participants' background and working environment. (2) The second section examined the definition of AKI and when the initiating of RRT was clinically indicated. (3) The third section looked at the availability of technology in different hospitals and sought the participants' views about different technical aspects of RRT (techniques and modality of RRT, type of anticoagulation, machines available for therapy) and explored specific questions about RRT management (RRT protocols, dose prescription, complications of RRT).

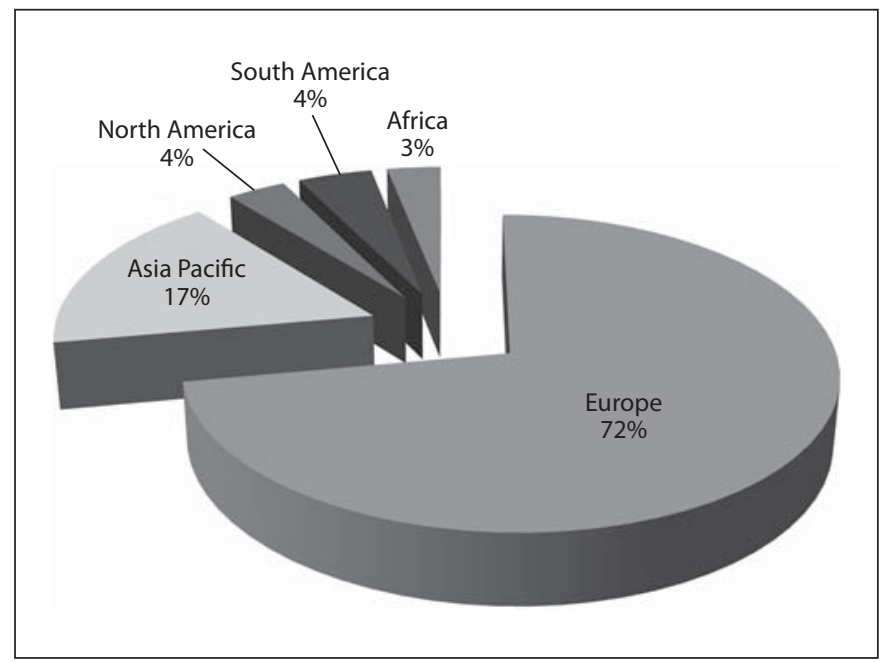

Fig. 1. Four hundred forty-four questionnaires were collected from 607 participants who came from Europe, Asia, Africa and America.

\section{Statistical Analysis}

All documents were analyzed with reference to a Microsoft Access database, which an independent pool of researchers had compiled from hardcopy sources. All data are presented either as absolute numbers or as a percentage of the examined questionnaires $(\mathrm{n}=440)$. Statistical analysis was performed using the SPSS 11.5 software package. The $\chi^{2}$ test was used to compare proportions. $\mathrm{p}<0.05$ was considered statistically significant.

\section{Results}

\section{Section 1}

Four hundred and sixty questionnaires were collected from 607 participants; of these, 440 (96\%) had been correctly completed and were therefore considered for the present analysis. Sixteen forms were excluded from analysis because more than $30 \%$ of the questions had not been answered. It should be mentioned that Europe continued to dominate greatly $(72 \%)$ but participation from the rest of the world (28\%) had increased significantly (fig. 1) compared to previous years $[13,14]$ and overall 5 continents and 58 nations were represented at the meeting. Specialties were quite evenly distributed, since $55 \%$ of responders were nephrologists and $45 \%$ intensivists. Academic and nonacademic centers participated in similar proportions: $56 \%$ of participants came from university hospitals or teaching hospitals, whereas $44 \%$ came from city or community hospitals. 


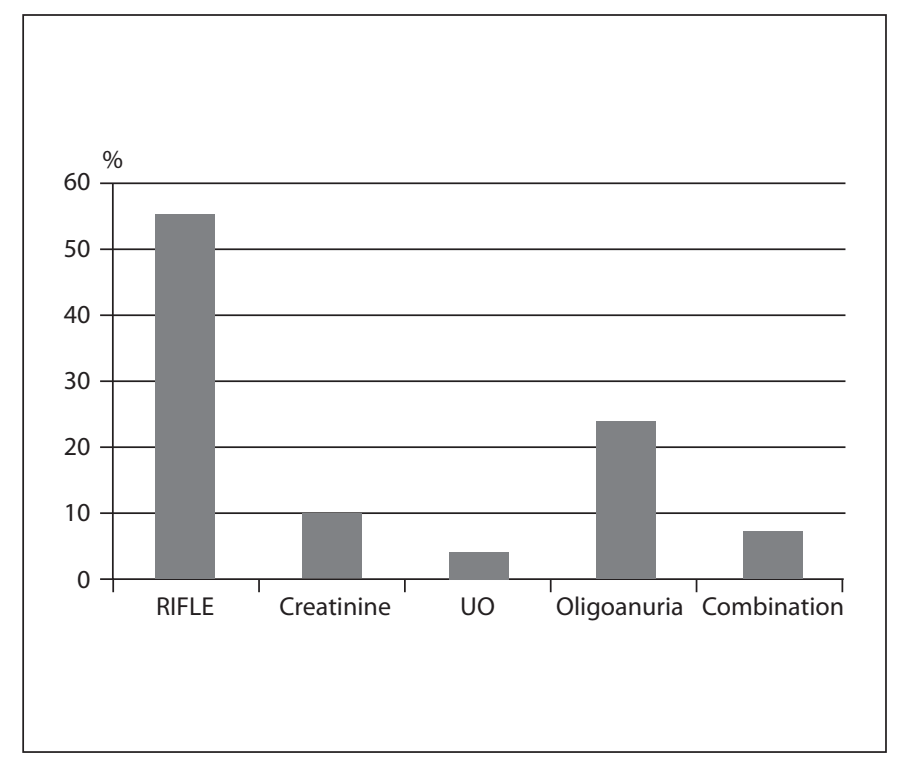

Fig. 2. Criteria used by participants to define AKI. Responders had the option to select more than one answer.

\section{Section 2}

Participants were asked to select one or more of four possible criteria to diagnose AKI: oligoanuria, increase in creatinine, decrease in urine output (UO) or RIFLE criteria. Interestingly, the most common answer was RIFLE criteria (55\%) followed by the presence of oligoanuria (24\%). Creatinine was selected by $10 \%$ of responders and $4 \%$ used the decrease in UO as a criterion (fig. 2). The remainder (7\%) selected a combination of the above answers giving 19 different definitions. The timing to start dialysis is an interesting and particularly complex topic: participants were asked to indicate which level of creatinine, diuresis, potassium and fluid overload triggered their decision to prescribe RRT in the ICU. Responders seemed to preferentially start dialysis within creatinine ranges of $2.3-3.4 \mathrm{mg} / \mathrm{dl}(28 \%)$ and $3.4-4.5 \mathrm{mg} / \mathrm{dl}(26 \%)$ and within blood urea nitrogen ranges of $29-67 \mathrm{mg} / \mathrm{dl}$ (14\%) and $68-115 \mathrm{mg} / \mathrm{dl}$ (37\%). Potassium levels to start extracorporeal treatment reached a high consensus around the range of 6-6.9 $\mathrm{mmol} / \mathrm{l}(51 \%)$. The majority of our participants would start RRT with a UO level of 150 $200 \mathrm{ml} / 12 \mathrm{~h}$ (43\%) or $100-150 \mathrm{ml} / 12 \mathrm{~h}$ (18\%). When fluid overload was analyzed, surprisingly, about $30 \%$ of responders indicated that they would prescribe dialysis only in case of severe fluid overload (requiring mechanical ventilation and/or causing impaired skin integrity),

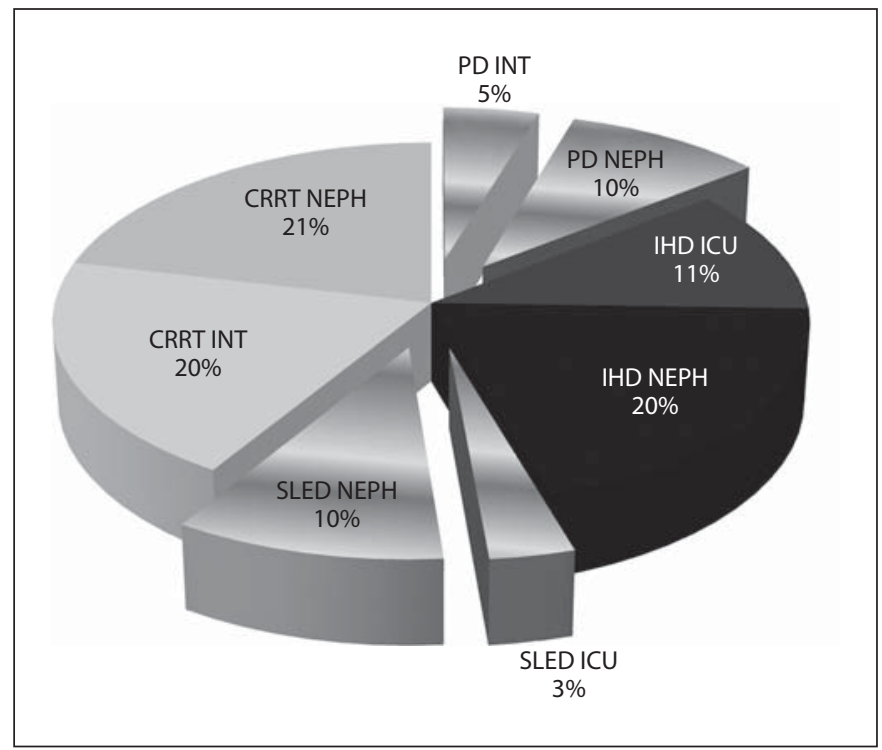

Fig. 3. Analysis of preferred RRT: peritoneal dialysis (PD), intermittent hemodialysis (IHD), CRRT, and sustained low-efficiency dialysis (SLED). The choices of intensivists (INT) and nephrologists (NEPH) tended to be similar as regards CRRT.

whereas another $25 \%$ would prescribe it in case of moderate to severe fluid overload and less than $20 \%$ of responders in case of mild to moderate fluid overload. Interestingly, $74 \%$ of answers to the question of indicating 'which of the above indications to begin RRT is the most common' remained blank, showing how the indication to start RRT is complex and often multifactorial. A wide agreement seemed to be present on the prescription of RRT for extended indications (i.e. sepsis), either isolated (54\%) and with a simultaneous presence of AKI (63\%).

\section{Section 3}

As far as the management of continuous RRT (CRRT) is concerned, $37 \%$ of responders admitted that there is no standardized protocol at their institution. Overall availability of techniques in different institutions was analyzed: absolute analysis of RRT techniques used showed that CRRT was preferred by most specialists (86\%), followed by intermittent hemodialysis (65\%) and sustained low-efficiency dialysis (28\%). Notably, peritoneal dialysis was considered by $30 \%$ of responders. It is interesting to note that there was a significant difference between intensivists and nephrologists (fig. 3): apparently, there is a similar preference for continuous techniques (CRRT) between the two groups, whereas the nephrologists tend to choose more frequently intermittent techniques (inter- 


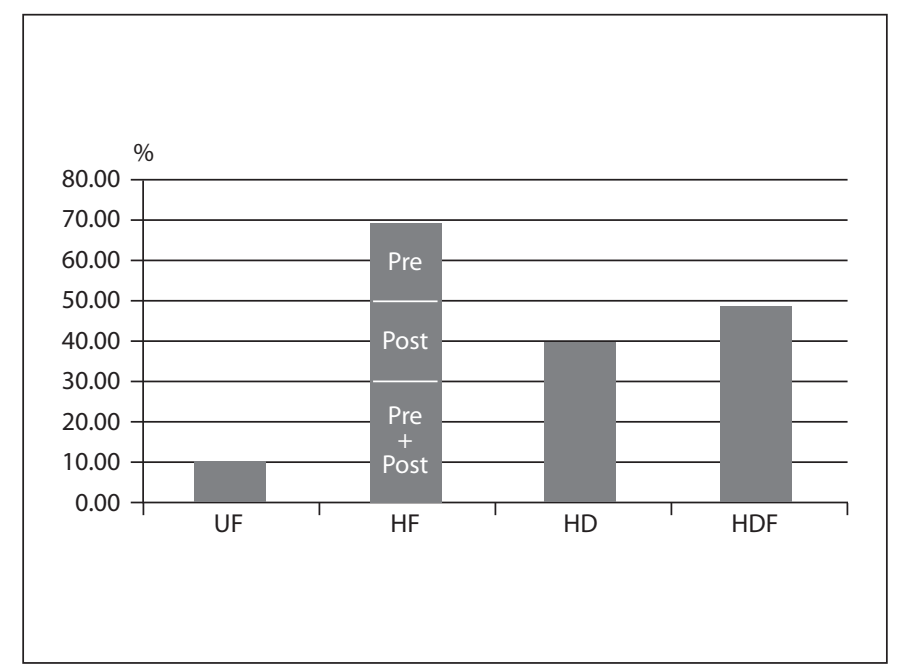

Fig. 4. Analysis of prevalently prescribed modalities: ultrafiltration (UF), predilution HF, postdilution HF, pre- and postdilution HF, hemodialysis (HD), and HDF. Pre = Predilution; Post $=$ postdilution.

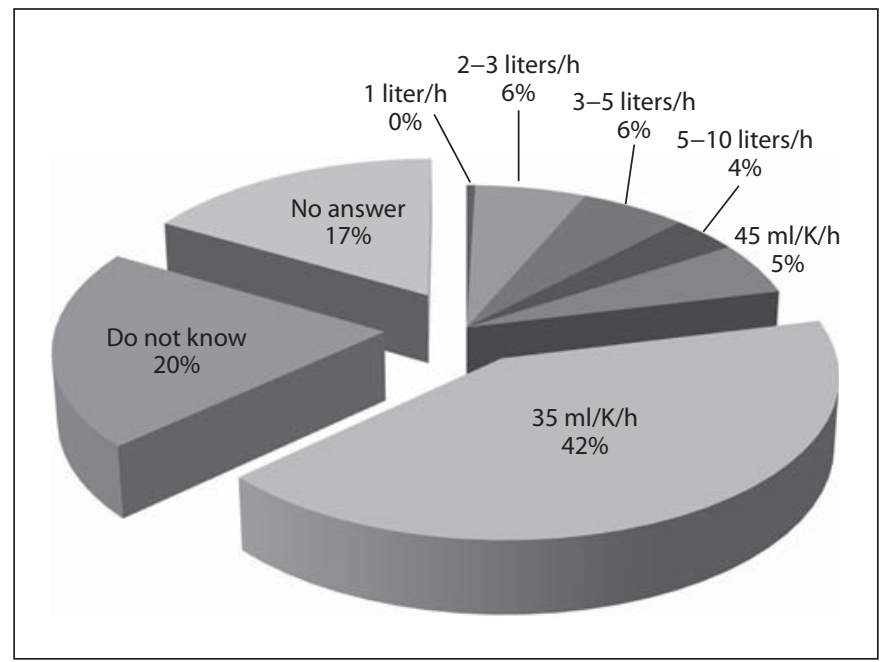

Fig. 5. Participants' view about dialysis efficiency targets $(\mathrm{K}=$ clearance) during RRT.

Table 1. Technical and clinical complications and concerns occurring frequently during RRT management

\begin{tabular}{lrlrlrrr}
\hline Technical complications & $\%$ & Clinical complications & $\%$ & Technical concerns & $\%$ & Clinical concerns & $\%$ \\
\hline Filter clotting & 74 & Hematoma & 4 & Use of heparin & 32 & Nursing workload & 40 \\
Low ultrafiltration & 4 & Thrombosis & 9 & Low efficiency & 5 & Blood loss \\
Access dysfunction & 32 & Hemorrhage/bleeding & 28 & Device costs & 23 & Multidisciplinary & 21 \\
Line disconnection & 1 & Hypotension & 37 & Staff problems & 21 & Unclear indications & 9 \\
Machine malfunction & 7 & Low efficiency & 9 & Machine requirements & 5 & Questionable benefits & 5 \\
Fluid balance errors & 4 & Related infection & 11 & Staff requirements & 11 & Induced infections & 7 \\
Nursing problems & 13 & Need to change treatment & 7 & Lack of standards & 7 & Other \\
Other & 1 & Hematoma & 4 & Frequent clotting & 22 & Nursing workload & 40 \\
\hline
\end{tabular}

mittent hemodialysis or sustained low-efficiency dialysis) and peritoneal dialysis $(\mathrm{p}<0.01)$. In particular, it was confirmed that the vast majority have different choices for the prescription of RRT at their disposal: in fact, only $34 \%$ of responders answered that they routinely use only one dialytic technique in their institution, whereas $31 \%$ have a choice between at least two techniques and $24 \%$ between three different dialytic techniques; apparently, $11 \%$ of our participants use all four forms of dialysis. Participants were then asked to specify their routinely used modality (or combinations of modalities) for blood purification. As depicted in figure 4, hemofiltration (HF) and hemodiafiltration (HDF) were the prevalent options, selected in 69 and $48 \%$ of cases, while hemodialysis (39\%) was the least frequent choice. Subgroup differences were significant $(\mathrm{p}<0.01)$, since intensivists apparently tended to preferentially prescribe $\mathrm{HF}$ and HDF, whereas the choice of nephrologists between HF, hemodialysis and HDF was more balanced.

Unfractionated heparin (whether at high or low doses) remained the preferred anticoagulant choice, having been selected by more than $80 \%$ of participants. Low-molecular-weight heparins or infusion of citrate were both selected in about one fifth of cases.

The following question concerned the participants' view about the prescription of RRT dose. The most frequently selected dosage was $35 \mathrm{ml} / \mathrm{kg} / \mathrm{h}$ (46\%) (fig. 5). A relatively high number of blank answers (17\%) and a large 
Table 2. Expected technical and clinical developments and future research into RTT management

\begin{tabular}{lrlrlrcr}
\hline Technical development & $\%$ & Clinical development & $\%$ & Technical research & $\%$ & Clinical research \\
\hline New vascular access & 8 & Reduced complications & 23 & Heparin-coated circuits & 11 & Staff training programs & 23 \\
Alternative anticoagulation & 26 & Improved standards & 10 & Heparin-free treatment & 29 & Extended indications & 15 \\
New membranes & 11 & Improved outcomes & 24 & New hemofilters & 8 & Improved efficiency & 9 \\
New filters & 9 & System reliability & 8 & New vascular access & 7 & Treatment of sepsis & 38 \\
New techniques & 14 & Replacement fluids & 11 & New machines & 5 & New biomaterials & 7 \\
New indications & 15 & Clinical stability & 18 & New techniques & 9 & Reduced complications & 9 \\
New machines & 16 & Other & 0 & Standardized protocols & 29 & & \\
Higher efficiency & 18 & & & Replacement fluids & 5 & \\
\hline
\end{tabular}

Table 3. Maximum filter duration either by protocol (if present) or by actual experience

\begin{tabular}{lrcr}
\hline $\begin{array}{l}\text { Average hours to } \\
\text { protocol filter change }\end{array}$ & $\%$ & $\begin{array}{l}\text { Average hours } \\
\text { of filter lifespan }\end{array}$ \\
\hline 12 & 14 & $<8$ & 41 \\
24 & 34 & $8-12$ & 32 \\
36 & 4 & $12-18$ & 13 \\
48 & 16 & $18-24$ & 11 \\
72 & 23 & $>24$ & 3 \\
\hline
\end{tabular}

Table 4. Dialysis catheter type and site

\begin{tabular}{lrll}
\hline Catheter type & $\%$ & Catheter site & $\%$ \\
\hline DL 13.5 F & 33 & Femoral & 51 \\
DL 12 F & 54 & Jugular & 63 \\
DL 11 F & 15 & Subclavian & 23 \\
SL & 5 & & \\
\hline
\end{tabular}

$\mathrm{DL}=$ Double lumen; $\mathrm{SL}=$ single lumen; $\mathrm{F}=$ French .

group of 'I do not know' answers (20\%) showed how this issue still needs further elaboration among operators in the field of critical RRT. Finally, responders' considerations of clinical and technical concerns, clinical and technical developments, clinical and technical research, average circuit lifespan, commonly used filter type and surface, dialysis catheter size and site are listed in tables 1-5. Technical and clinical issues occurring during RRT seemed to have remained similar to 2004 .
Table 5. Filter surface and type

\begin{tabular}{lclr}
\hline Filter surface & $\%$ & Filter type & $\%$ \\
\hline $1 \mathrm{~m}^{2}$ & 15 & Cellulose & 3 \\
$1.5 \mathrm{~m}^{2}$ & 36 & Polysulfone & 60 \\
$2 \mathrm{~m}^{2}$ & 13 & PAN & 8 \\
Depends on prescription & 34 & PMMA & 4 \\
Other & 2 & AN69 & 29 \\
\hline
\end{tabular}

PAN = Polyacrylonitrile; PMMA = polymethylmethacrylate; AN69 = acrylonitrile and sodium methallyl sulfonate.

\section{Discussion}

We took advantage of an international meeting on CRRT and critical care nephrology (Third Critical Care Nephrology International Survey, held in Vicenza, Italy, in 2007) to investigate the clinicians' view about AKI management by means of a questionnaire. The group of participants was quite large $(n=444)$ and a broad distribution was evident. A significant improvement was shown regarding questions on the definition of AKI in comparison to 2004: the use of RIFLE significantly increased (from 16 to 55\%) and the number of different combined definitions decreased accordingly (from about 200 to 19) [13]. In 2007, RIFLE criteria were chosen by the absolute majority of the participants and only few of them still required a personalized definition. This finding was not surprising in the light of increasing reports in the literature of the adult and pediatric patients critically ill with AKI. The indication to start RRT was more thoroughly investigated compared to previous surveys: our responders seem to prescribe dialysis relatively early, when low to medium levels of creatinine and urea are 
reached. What may look surprising, however, is the fact that very low levels of UO or severe fluid overload were preferentially selected as an indication for starting RRT: the great impact of fluid balance on the mortality of AKI patients might have been underestimated in the last years and it will probably be reevaluated in the near future in the light of recent observational papers $[15,16]$. As far as nonrenal indications are concerned, 54\% of responders stated that they (conceptually) agree with nonrenal indications. The lack of scientific evidence is usually the main reason for skepticism about adopting extracorporeal treatment; nonetheless, a fair amount of responders declared that they would start RRT in case of septic shock even in the absence of AKI. This contradiction was, however, less evident than in 2004, when about $90 \%$ of clinicians declared that they agreed with RRT for nonrenal indications. An analysis of techniques available in different institutions showed a certain prevalence of continuous techniques. Nevertheless, it was shown that different techniques were available in about $66 \%$ of institutions. As shown in our survey 4 years earlier, a large number of the participants seemed uncertain about prescribed treatments: participants usually claimed that they prescribe a dose of $35 \mathrm{ml} / \mathrm{kg} / \mathrm{h}$ (46\%) but more than one third of those that responded did not provide an explicit answer to this question. It must be said, however, that the number of unclear responses to this question was significantly higher in 2004 (54\% of questions unanswered) and the dose of $35 \mathrm{ml} / \mathrm{kg} / \mathrm{h}$ was then selected by only $25 \%$ of people [13]. As a matter of fact, unfractionated heparin infusion was still the preferred anticoagulation technique, followed by low-molecular-weight heparin and citrate. Anticoagulation side effects (bleeding and filter clotting) are still a cause for complaint. Interestingly, the other technical and clinical issues and complaints which occur during RRT still seem to be similar to those in 2004, maybe showing that there has been little improvement in the technical aspects of RRT during this relatively short period of time.

Finally, some of the limitations of the present survey need to be remarked upon: the delegates who attended this meeting were obviously a self-selected population group and their answers cannot reasonably reflect the worldwide daily reality of patient care, because there is clearly a European bias in our results. As a matter of fact, the answers of the participants do not necessarily completely reflect the actual clinical practice worldwide and they might represent a sample of 'expert operators' in the field of RRT. Moreover, these results should be viewed as a collection of opinions and thoughts rather than an accurate description of clinical practice. Also, one must take into account that, even if we explicitly asked responders to provide their personal opinions, they have been trained during the course and their views might have been modified. Finally, in a relatively small (but also unquantifiable) number of cases, participants might have simply been providing answers as though they were completing a (right or wrong) test instead of communicating their personal experience. Nevertheless, it must be said that participants were invited to fill in the questionnaire on a voluntary basis, and they were explicitly asked to frankly and freely state their personal opinions. Furthermore, this is the third time we have submitted this questionnaire in the last 12 years: the analyzed answers show coherent and clear trends towards a homogeneous modification of the opinions of those who participated, and these responses reliably reflect some of the changes that have taken place in real-world clinical practice.

\section{Conclusion}

Our results must be seen in the light of a self-selected, European-biased survey. Nevertheless, we considered it a unique opportunity to interview a large number of specialized operators in the field of critical care nephrology. Our survey confirmed an evident trend towards a standardization of the AKI definition: the RIFLE criteria seem to be showing a clinical impact on daily practice. A trend towards increased awareness about RRT dosage seems evident when compared with previous surveys, even if there are still no definitive indications for RRT and no guidelines about prescribing RRT among practitioners in the field of critical care nephrology.

\section{Disclosure Statement}

The authors declare that they have no competing interests. 


\section{References}

1 Bellomo R, Ronco C, Kellum A, Mehta RL, Palevsky P, ADQI workgroup: Acute renal failure - definition, outcome measures, animal models, fluid therapy and information technology needs: the Second International Consensus Conference of Acute Dialysis Quality Initiative (ADQI) group. Crit Care 2004;8:R204-R212.

2 ADQI: Acute Dialysis Quality Initiative. http://www.adqi.net (accessed May 2010).

$\checkmark 3$ Mehta RL, Kellum JA, Shah SV, Molitoris BA, Ronco C, Warnock DG, Levin A, Acute Kidney Injury Network: Acute Kidney Injury Network: report of an initiative to improve outcomes in acute kidney injury. Crit Care 2007;11:R31.

4 Ricci Z, Cruz D, Ronco C: The RIFLE criteria and mortality in acute kidney injury: a systematic review. Kidney Int 2008;73:538-546.

5 VA/NIH Acute Renal Failure Trial Network, Palevsky PM, Zhang JH, O’Connor TZ, Chertow GM, Crowley ST, Choudhury D, Finkel K, Kellum JA, Paganini E, Schein RM, Smith MW, Swanson KM, Thompson BT, Vijayan A, Watnick S, Star RA, Peduzzi P: Intensity of renal support in critically ill patients with acute kidney injury. N Engl J Med 2008;359:7-20.

6 RENAL Replacement Therapy Study Investigators, Bellomo R, Cass A, Cole L, Finfer S, Gallagher M, Lo S, McArthur C, McGuinness S, Myburgh J, Norton R, Scheinkestel C, $\mathrm{Su}$ S: Intensity of continuous renal-replacement therapy in critically ill patients. N Engl J Med 2009;361:1627-1638.
7 Vesconi S, Cruz DN, Fumagalli R, KindgenMilles D, Monti G, Marinho A, Mariano F Formica M, Marchesi M, René R, Livigni S Ronco C, DOse REsponse Multicentre International collaborative Initiative (DO-RE-MI Study Group): Delivered dose of renal replacement therapy and mortality in critically ill patients with acute kidney injury. Crit Care 2009; 13:R57.

-8 Uchino S, Doig GS, Bellomo R, Morimatsu H, Morgera S, Schetz M, Tan I, Bouman C, Nacedo E, Gibney N, Tolwani A, Ronco C, Kellum JA, Beginning and Ending Supportive Therapy for the Kidney (B.E.S.T. Kidney) Investigators: Diuretics and mortality in acute renal failure. Crit Care Med 2004;32: 1669-1677.

9 Uchino S, Kellum JA, Bellomo R, Doig GS, Morimatsu H, Morgera S, Schetz M, Tan I, Bouman C, Macedo E, Gibney N, Tolwani A, Ronco C, Beginning and Ending Supportive Therapy for the Kidney (BEST Kidney) Investigators: Acute renal failure in critically ill patients: a multinational, multicenter study. JAMA 2005;294:813-818.

10 Uchino S, Bellomo R, Kellum JA, Morimatsu H, Morgera S, Schetz MR, Tan I, Bouman C, Macedo E, Gibney N, Tolwani A, OudemansVan Straaten HM, Ronco C, Beginning and Ending Supportive Therapy for the Kidney (B.E.S.T. Kidney) Investigators Writing Committee: Patient and kidney survival by dialysis modality in critically ill patients with acute kidney injury. Int J Artif Organs 2007;30:281-292.

-11 Uchino S, Bellomo R, Morimatsu H, Morgera S, Schetz M, Tan I, Bouman C, Macedo E, Gibney N, Tolwani A, Oudemans-van Straaten H, Ronco C, Kellum JA: Continuous renal replacement therapy: a worldwide practice survey. The beginning and ending supportive therapy for the kidney (B.E.S.T kidney) investigators. Intensive Care Med 2007;33:1563-1570.
12 Srisawat N, Lawsin L, Uchino S, Bellomo R, Kellum JA, BEST Kidney Investigators: Cost of acute renal replacement therapy in the intensive care unit: results from The Beginning and Ending Supportive Therapy for the Kidney (BEST Kidney) Study. Crit Care 2010; 14:R46

13 Ricci Z, Ronco C, D’Amico G, De Felice R, Rossi S, Bolgan I, Bonello M, Zamperetti N, Petras D, Salvatori G, Dan M, Piccinni P: Practice patterns in the management of acute renal failure in the critically ill patient: an international survey. Nephrol Dial Transplant 2006;21:690-696.

14 Ronco C, Zanella M, Brendolan A, Milan M, Canato G, Zamperetti N, Bellomo R: Management of severe acute renal failure in critically ill patients: an international survey in 345 centres. Nephrol Dial Transplant 2001; 16:230-237.

15 Bouchard J, Soroko SB, Chertow GM, Himmelfarb J, Ikizler TA, Paganini EP, Mehta $\mathrm{RL}$, Program to Improve Care in Acute Renal Disease (PICARD) Study Group: Fluid accumulation, survival and recovery of kidney function in critically ill patients with acute kidney injury. Kidney Int 2009;76:422-427.

16 Payen D, de Pont AC, Sakr Y, Spies C, Reinhart K, Vincent JL, Sepsis Occurrence in Acutely Ill Patients (SOAP) Investigators: A positive fluid balance is associated with a worse outcome in patients with acute renal failure. Crit Care 2008;12:R74 